\title{
Atomistic Dynamics of a Viral Infection Process: Release of Membrane Lytic Peptides from a Non-Enveloped Virus
}

\author{
Short Title: Atomistic Dynamics of a Viral Infection Process
}

\author{
Asis K. Jana, Eric R. May \\ Department of Molecular and Cell Biology, University of Connecticut, Storrs, CT 06269 \\ Corresponding Author: Eric May, eric.may@uconn.edu \\ Keywords: All-Atom Molecular Dynamics Simulations, Viral Capsid, Endosomal Escape, \\ Enhanced Sampling
}

\begin{abstract}
Molecular simulations have played an instrumental role in uncovering the structural dynamics and physical properties of virus capsids. In this work we move beyond equilibrium physicochemical characterization of a virus system to study a stage of the infection process which is required for viral proliferation. Despite many biochemical and functional studies, the molecular mechanism of host cell entry by non-enveloped viruses remains largely unresolved. Flock House Virus (FHV) is model system for non-enveloped viruses and is the subject of the current study. FHV infects through the acid-dependent endocytic pathway, where low $\mathrm{pH}$ triggers externalization of membrane disrupting $(\gamma)$ peptides from the capsid interior. Employing all-atom equilibrium and enhanced sampling simulations, the mechanism and energetics of $\gamma$ peptide liberation and the effect of $\mathrm{pH}$ on this process is investigated. Our computations agree with experimental findings and reveal nanoscopic details regarding the $\mathrm{pH}$ control mechanism which are not readily accessible in experiments.
\end{abstract}




\section{Introduction}

Understanding the molecular mechanism by which viruses invade host cells can provide insights for developing anti-viral therapies and drug treatments for diseases caused by these viruses. Enveloped viruses such as human immunodeficiency virus (HIV), Ebola and Influenza breach host cell membranes through a membrane-fusion process catalyzed by fusion proteins in the viral envelope and this process has become increasingly well characterized. $(1,2)$ However, the mechanisms of cellular membrane penetration by non-enveloped viruses remain enigmatic. Emerging studies suggest that several of these non-enveloped viral families including, Picornaviridae, Polyomaviridae, Reoviridae, Parvoviridae, Adenoviridae, and Nodaviridae contain a membrane active component of their capsid, which becomes active under endosomal conditions.(3-6)

Flock House Virus (FHV), an insect infecting member of the Nodaviridae family serves as model system for investigating the molecular details of cell entry in non-enveloped viruses.(7) FHV can replicate in insects(8), plants(9) and yeast cells(10) and the viral genome consists of two single-stranded positive-sense RNA genomes. The capsid proteins initially assemble into an immature $T=3$ icosahedral capsid, composed of 60 identical icosahedral asymmetric units (iASU) and each iASU consist of three chemically identical $\alpha$ subunits, which have 407 residues. The subunits are arranged in three slightly different quasi-equivalent positions (A, B and C). The capsid undergoes maturation to an infectious particle involving autoproteolytic cleavage of the capsid proteins between residues Asn363 and Ala364, catalyzed by Asp75. This results in two particleassociated cleavage products, a mature capsid protein $\beta$ (residues 1-363) and a 44 residue $\gamma$ peptide (residues 364-407). The cleavage site (N363/A364) is on the interior of the capsid shell and 
mutations to either the catalytic D75 residue or the cleaved residue N363, block the maturation cleavage and render the particles non-infectious.(11) The implication is that a dissociated $\gamma$ peptide is essential for viral infection.

The X-ray crystal structure of the mature capsid was resolved at $2.7 \AA$ resolution and shows that $\gamma$ peptides are non-covalently associated with the capsid interior (Figure 1). While the Nterminal residues (364-385) of $\gamma$ form an amphipathic $\alpha$-helix. , the predominantly hydrophobic Cterminal region of $\gamma$ (residues 385-407) are not resolved in the crystal structure, presumably do to a disordered structure and/or conformational heterogeneity across the capsid.(12) Biophysical studies have shown the N-terminal helical region (termed $\gamma_{1}$ ) is capable of membrane disruption(13-15) while the C-terminal region of $\gamma$ plays an important role for specific recognition of the FHV genome and packaging of genomic RNA during assembly.(16) Subsequent studies showed that the full-length peptide has significantly greater membrane lytic activity than $\gamma_{1}$ and mutations and deletions in the C-terminal region could impair the lytic activity and infectivity. (17, 18) The position of the helices of $\gamma$ are located in significantly different environments in the three subunits of the iASU due to quasi-equivalence. The A subunits form pentamers and the $\gamma$ peptides from the A subunits $\left(\gamma_{\mathrm{A}}\right)$ interact with each other and form a pentameric helical bundle with the helical axes oriented normal to the capsid surface (Figure 1C). This organization is stabilized by hydrophilic interactions between peptides and are positioned far from the viral RNA.(19) In contrast, $\gamma$ peptides from $\mathrm{B}$ and $\mathrm{C}$ subunits are adjacent to the resolved viral RNA segments and form numerous contacts with the genome. $(19,20)$ Based on the orientation of the $\gamma_{\text {A }}$ peptides and lack of RNA contacts, it has been speculated that the pentameric helical bundle of $\gamma_{\mathrm{A}}$ peptides are responsible for membrane lysis. $(19,21)$ This model is supported by studies on Nudaurelia capensis $\omega$ virus $(\mathrm{N} \omega \mathrm{V}, T=4)$, where $\gamma$ peptides are positioned in similar environments (quasi- 
equivalent).(22) Studies with $\mathrm{N} \omega \mathrm{V}$ virus using liposome-based assays show that maximal membrane disruption is acquired with less than half of the subunits of the capsid cleaved,(22) whereas structural studies using time-resolved cryoelectron microscopy (cryo-EM) suggest that subunits surrounding the icosahedral fivefold axis (A subunits) are cleaved much earlier than the subunits positioning in different quasi-equivalent environment of the capsid.(23)

While the FHV capsid crystal structure shows that $\gamma$ peptides are sequestered inside the capsid,(12) it is believed that the role of the $\gamma$ peptides in the infection process is to disrupt endosomal membranes and allow the capsid to escape these membrane bound compartments. The proposed model is that low $\mathrm{pH}$ induces conformational and/or dynamic changes to the capsid which allow for or promote $\gamma$ peptides to be externalize from the capsid. This model is supported by several lines of evidence. i) Mass spectrometry combined with limited proteolysis suggest 'occasional breathing' or transient exposure of $\gamma$ peptides on the viral surface.(24) ii) Liposome dye leakage assays showed a $\mathrm{pH}$ dependent lytic activity of FHV capsids, with maximal leakage at $\mathrm{pH}$ 6.0, consistent with the $\mathrm{pH}$ of endosomes.(21) iii) FHV infectivity was also correlated to endosomal $\mathrm{pH}$, as increasing the endosomal $\mathrm{pH}$ through the addition of bafilomycin $\mathrm{A} 1$, an $\mathrm{H}^{+}$
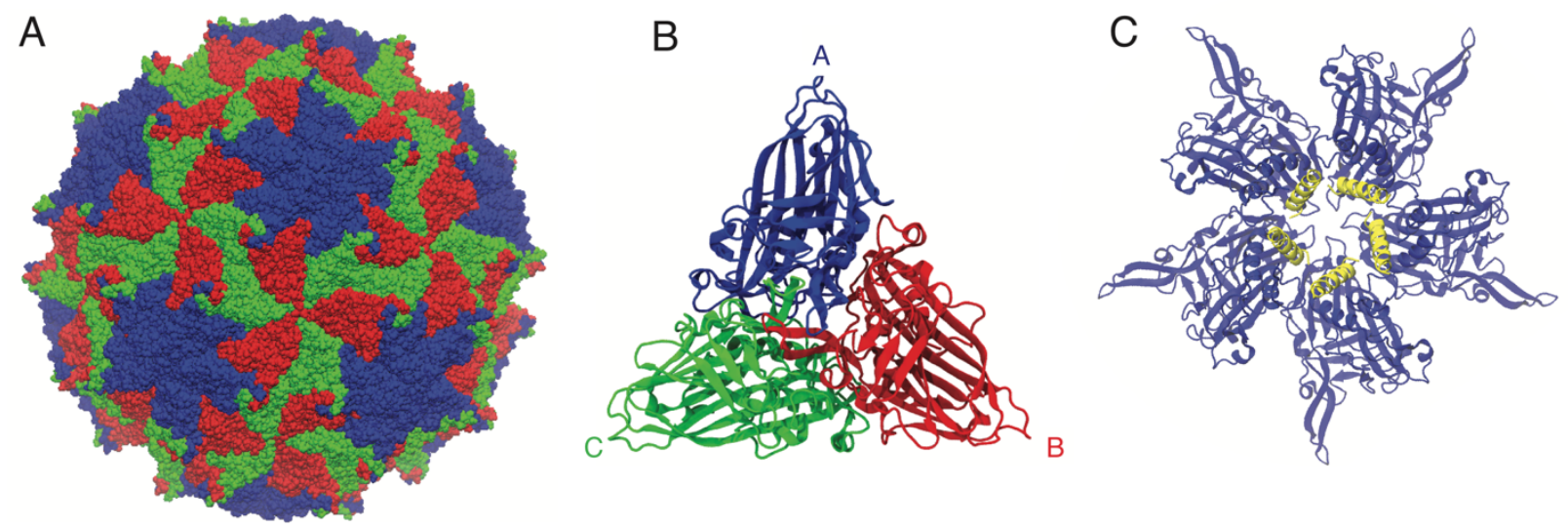

Figure 1. FHV structure. A) $T=3$ capsid structure. B) Asymmetric unit. C) Interior view of fivefold vertex. The A-proteins are blue and the yellow helices are the $\gamma$ peptides. All images are generated from PDBID: 4FTB. 
ATPase inhibitor, decreased infectivity.(21) iv) When liposome dye leakage assays were performed at $\mathrm{pH} 7.0$ following preincubation at $\mathrm{pH}$ 6.0, for as little as one hour, the dye leakage activity was equivalent to when the leakage assay was performed at $\mathrm{pH}$ 6.0.(21) v) bis-ANS, a fluorophore which binds aromatic and hydrophobic residues, had maximal binding at $\mathrm{pH}$ 6.0.(21)

While these previous findings provide strong support that FHV has an acid-dependent membrane disruption mechanism involving capsid conformational changes, the nature of structural transition and molecular details are not clearly understood. All-atom molecular dynamics (MD) simulations, sometimes termed a "computational microscope"(25, 26), has emerged as a powerful tool for studying viral systems, as this technique can provide significant insight into the structure and dynamics at a high spatial and temporal resolution, which is typically inaccessible in experiments. Furthermore, MD simulations can go beyond observation of structural features and thermodynamic quantities can be obtained, which can allow a structure-function model to be developed or validated. With ever increasing computational power, several simulation studies have been performed on full virus capsids to investigate the capsid dynamics and physicochemical properties.(27-30) In this present study we use fully atomistic MD simulation to investigate the details of a stage of a viral infection process: the liberation of $\gamma$ peptides from the interior of a non-enveloped capsid and the effects of $\mathrm{pH}$. Previous simulation studies have examined viral life cycle processes, namely assembly(31-36) and maturation(37-40) using various forms of coarse-grained models and/or implicit solvent models. We believe the current study provides a significant advance in the field by examining a viral infection process and its extensive use of atomistic simulations of a large portion of the capsid in explicit solvent through both equilibrium and enhanced sampling simulation methods. 


\section{Results}

\section{pH Effects on the Structure and Equilibrium Dynamics of FHV Capsid}

In our study, we begin by analyzing equilibrium simulations of the FHV pentameric capsid system, (Figure 2A) at neutral and low pH. These systems were simulated in triplicate for $500 \mathrm{~ns}$ each (see Table 1). The systems were relatively stable (as measured by Ca RMSD) during this time scale and similar levels of structural relaxation were observed across the independent trails for each $\mathrm{pH}$, Figure S1. The RMSD differences between the neutral and low $\mathrm{pH}$ systems are modest, but the low $\mathrm{pH}$ system displays a statically significant greater deviation than neutral $\mathrm{pH}$. The averaged RMSD values over the final $300 \mathrm{~ns}$ and averaged over the three trails are 0.385 $( \pm 0.05) \mathrm{nm}$ and $0.411( \pm 0.15) \mathrm{nm}$ for neutral and low $\mathrm{pH}$, respectively. We next analyzed the exposure of hydrophobic residues on the capsid surface through SASA calculations. It is experimentally known from bis-ANS fluorescence measurements that low $\mathrm{pH}$ induces increased
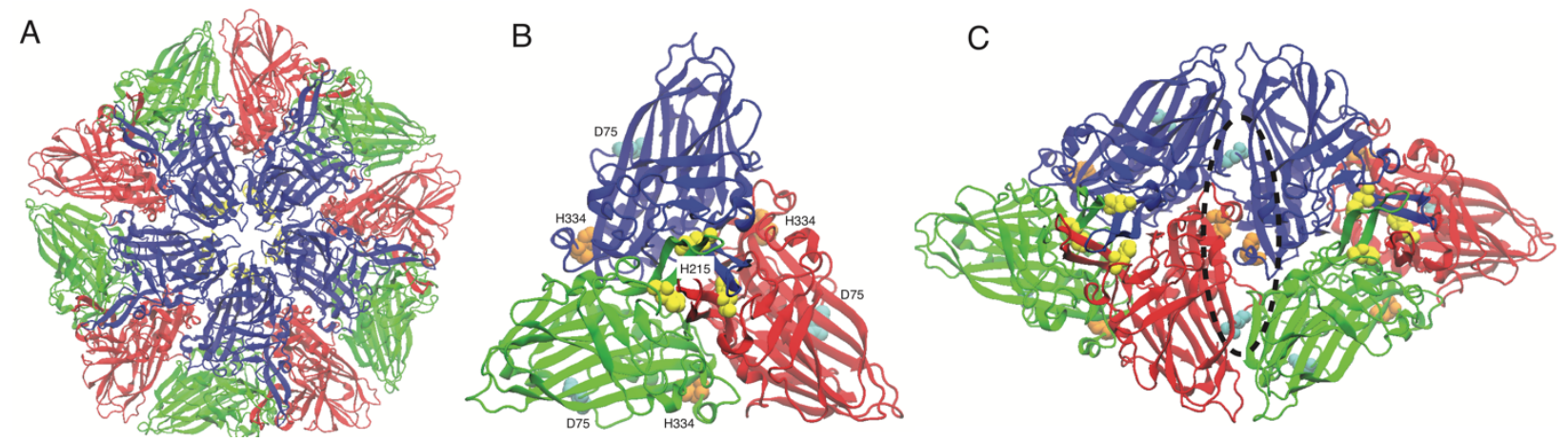

Figure 2. FHV simulation system and titratable residues. A) The simulation system consists of five asymmetric units surrounding a fivefold symmetry axis. The $\gamma$ peptides associated with the A subunits are retained and can be faintly seen in yellow beneath the fivefold vertex. B-C) Location of residues which change protonation states in low $\mathrm{pH}$ model. The residues consist of D75 (cyan), H215 (yellow) and H334 (orange) which are shown in vdW representation. Both a single asymmetric unit (B) and two adjacent asymmetric units (C) are shown to illustrate the location of titrated residues related to neighboring proteins. The $\mathrm{H} 215$ residues lie at the center of the asymmetric unit (pseudo threefold symmetric axis), while H334 and D75 lie at the interface between asymmetric units, which is highlighted by the dashed black line. All images are generated in an external to the capsid perspective (looking down on capsid), and are from PDBID: 4FTB. 
hydrophobic exposure(21), therefore we wanted to observe if our simulations captured this feature.

In Figure 3A the distributions of solvent accessible surface areas of hydrophobic residues are presented and it is observed that at low $\mathrm{pH}$ there is an increase in hydrophobic exposure.

While the SASA and RMSD calculations indicate increased conformational changes in the capsid at low $\mathrm{pH}$ we wanted to further connect structural changes to potential functional motions of the capsid. Based upon the structural organization of the $\gamma$ peptides it has been proposed in the literature that $\gamma \mathrm{s}$ will externalize through the fivefold symmetry axis, $(19,41)$ and it has been shown in $\mathrm{N} \omega \mathrm{V}$ that pentameric $\gamma \mathrm{s}$ are primarily responsible for membrane disruption.(22) Therefore, we examined the dynamics of the capsid at the fivefold symmetry axis to evaluate if low $\mathrm{pH}$ induces increased dynamics in the fivefold vertex region. The pore radius at the fivefold vertex was measured during the simulations and the profiles (Figure 3B,D) show a consistent increase in pore radius at low $\mathrm{pH}$, which is consistent across the multiple trials. The maximum observed pore radius at low $\mathrm{pH}$ is $7.3 \AA$ (Figure $3 \mathbf{E}$ ) which is more than double the maximum pore radius at neutral pH (3.5 A, Figure 3C). Furthermore, averaging over the last 300 ns and over the replicates shows the average pore radius at low $\mathrm{pH}$ is $4.0( \pm 0.6) \AA$, while the neutral $\mathrm{pH}$ simulations display

A

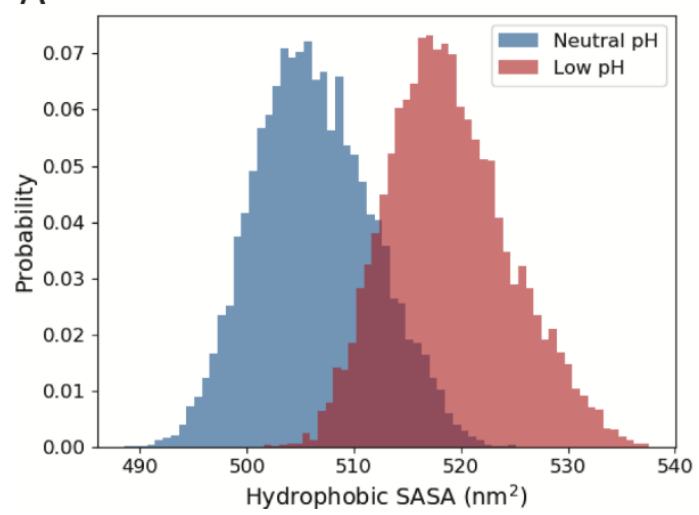

B

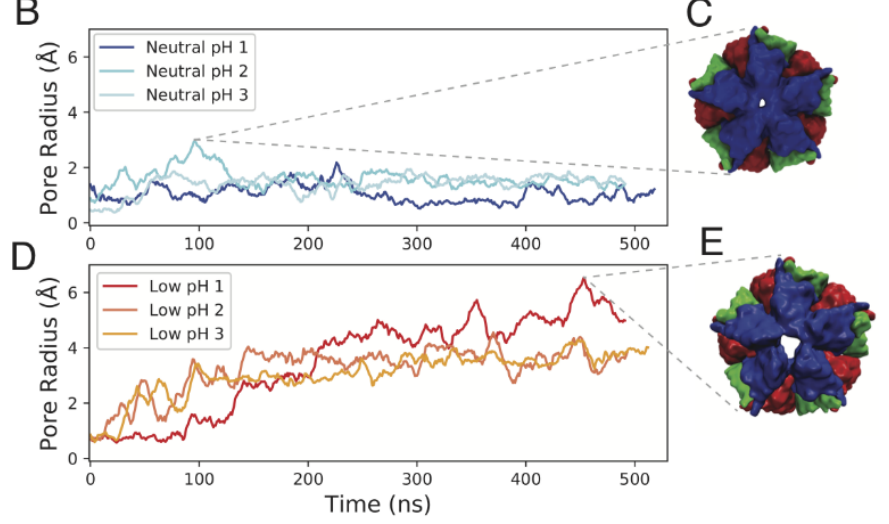

Figure 3. Structural changes induced by low pH A) The exposure of solvent accessible hydrophobic residues at neutral and low $\mathrm{pH}$. B-E) The pore radius at the fivefold axis is measured for the replicates at both neutral (B) and low $\mathrm{pH}(\mathrm{D})$. The structure with the maximum pore radius is shown for neutral $(\mathrm{C})$ and low $\mathrm{pH}(\mathrm{E})$ simulations. 
an average pore radius of only $1.3( \pm 0.2) \AA$. Representative equilibrium trajectories are visualized in Movie S1 (neutral $\mathrm{pH}$ ) and Movie S2 (low $\mathrm{pH}$ ) to demonstrate the fivefold pore dynamics. These pore measurements reveal a potential $\gamma$ liberation pathway through the fivefold axis and this pathway is consistent with the previous hypothesis based on the FHV structure-based model.

While we observe structural changes at the fivefold axis, which we believe are functionally significant, the residues which have undergone protonation at low $\mathrm{pH}$ are distal to the fivefold vertex (Figure 2B). To identify correlations between the titratable residues and capsid functional motions, we examined the structural dynamics of the systems by calculating secondary structural (SS) changes and linear mutual information. In Figure 4A we present the change in secondary structure of the A subunits relative to the neutral $\mathrm{pH}$ capsid, collected over the last $300 \mathrm{~ns}$ of the simulation trajectories. In Figure 4B-C, we show the location of residues with significant ( $>10$ $\%$ ) changes in SS. The residues which display significant changes in $\alpha$-helix or $\beta$-sheet content are 91-97, 169-171, 178, 203-204, 210-211, 244, 328-332, 334-335, 352-353 and 357-358. We see that these residues are proximal to the two histidine residues (H215 and H334) and participate in the protein-protein interfacial contacts. These subtle conformational changes could have a profound effect on the collective dynamics of the capsid. Furthermore, it appears there is a connection of residues, emanating from H334, which display differential SS changes and propagating toward the fivefold vertex (Figure 4C). Interestingly, we did not find any change in $\mathrm{SS}$ in the neighboring regions of the other protonated residue, D75.

We performed linear mutual information (LMI) analysis to characterize the correlations inside the capsid and the corresponding matrices are shown in Figure 4D-E(42, 43). In general, more mutual information is observed across many different regions of capsid at neutral $\mathrm{pH}$. Interestingly, there is significant mutual information between the inner most ("gate-keeper") loop 
of the fivefold axis with different regions of the capsid, but these correlations are diminished at low $\mathrm{pH}$. The region around $\mathrm{H} 215$ also show strong correlations across the capsid, including with the gate-keeper loop and again most of those correlations are diminished at low $\mathrm{pH}$. $\mathrm{H} 215$ is located at the center of the asymmetric unit (pseudo threefold axis, Figure 2B) and may serve as a hub residue capable of modulating protein-protein dynamics throughout in the capsid. Our results lead us to hypothesize that under low $\mathrm{pH}$ conditions the capsid motion of the fivefold axis is not
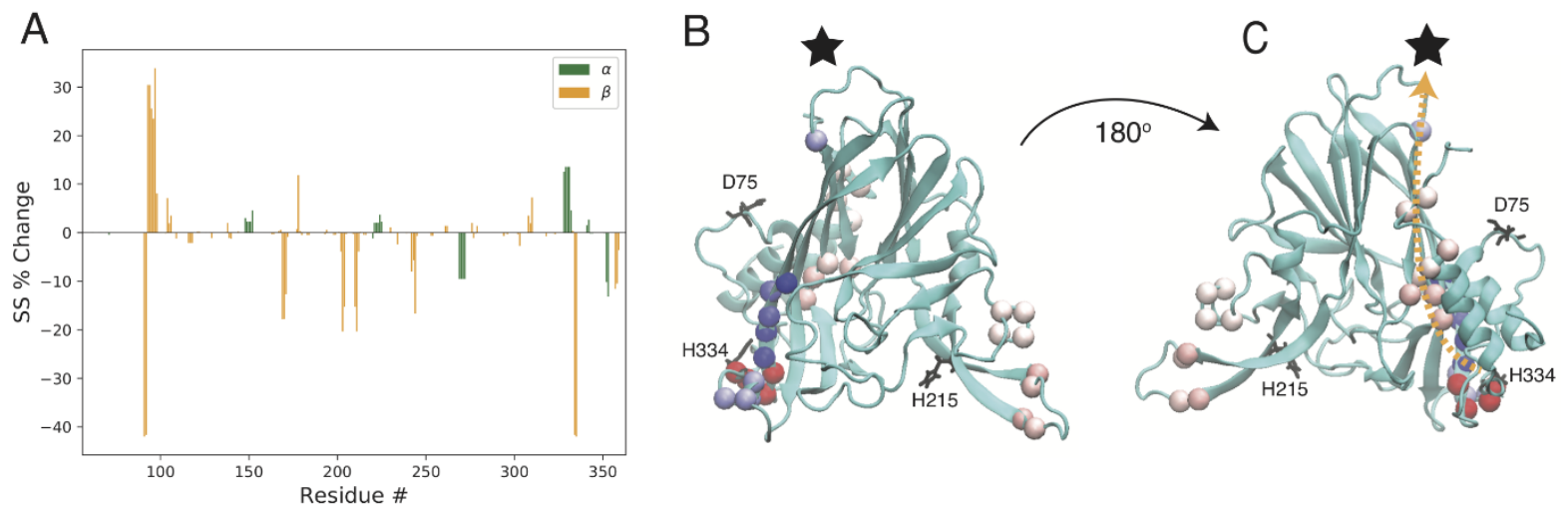

D
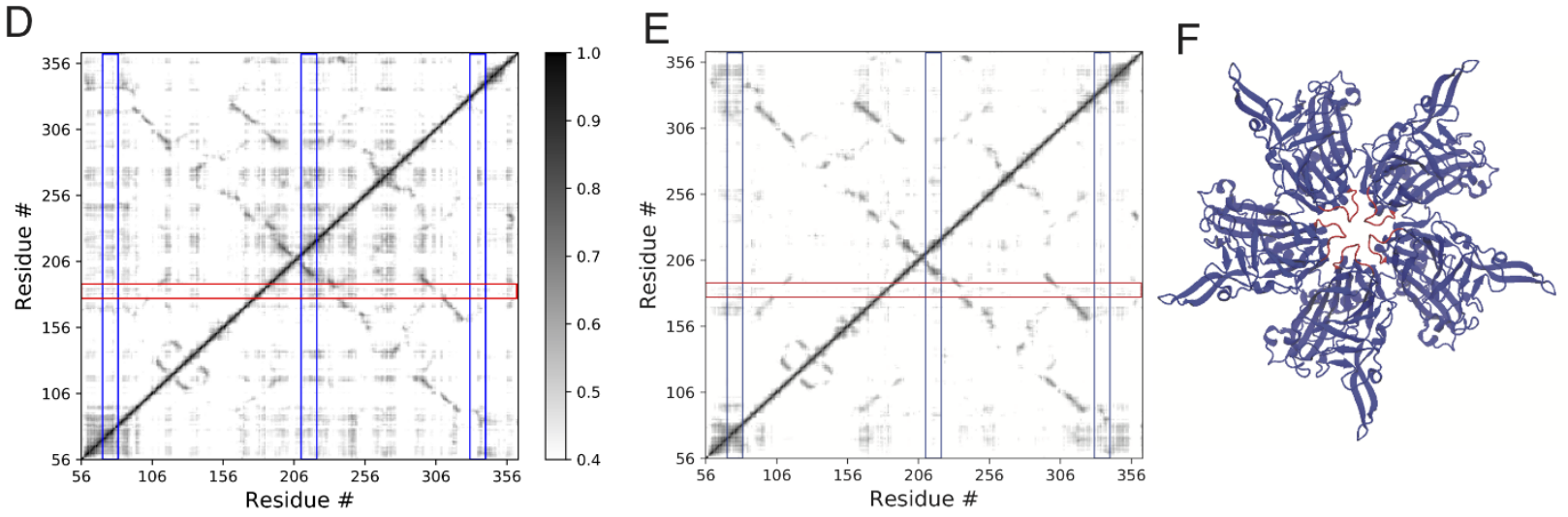

Figure 4. pH effects and dynamic couplings. A) The change in secondary structure (SS) in the A subunit in going from neutral to low $\mathrm{pH}$. B-C) The $\mathrm{C} \alpha$ atoms of residues with SS changes greater than $10 \%$ are displayed as vdW spheres on the A subunit structure. Colors scale is from red (largest decrease) to blue (largest increase). Top down (B) and internal (C) views are presented. The black star indicates the location of the fivefold vertex, and the orange dashed line is drawn to illustrate a pathway from $\mathrm{H} 334$ to the fivefold vertex. Residues which undergo protonation changes are labeled. D-E) The linear mutual information at neutral (D) and low $\mathrm{pH}$ (E). The correlations with less than 0.4 are set to zero to highlight stronger correlations. The blue vertical boxed regions are surrounding the titrating residues (D74, H215 and H334) and the red horizontal box is around residues 178-186, which is a loop at the fivefold vertex. F) The boxed red region in (D-E) corresponds to the red loop shown on the pentameric structure. 
concerted with the other regions of the capsid, which we suspect may lead to pore formation at the fivefold vertex. Our interpretation of the LMI and SS analyses is that the capsid is more connected and possibly more rigid at neutral $\mathrm{pH}$, and at low $\mathrm{pH}$ the protonation of the histidine residues leads to a disruption of the network. In particular these results point toward a model in which under low $\mathrm{pH}$ conditions the capsid motion of the fivefold axis is not concerted with the other regions of the capsid, which we suspect leads to weaker/softer interactions at the fivefold vertex that is supported by the pore analysis (Figure 3B-E) and further investigated in the following sections.

\section{$\gamma$ liberation pathways from Non-Equilibrium Simulations}

To gain insight into the molecular details of $\gamma$ liberation, we performed biased (steered MD) non-equilibrium simulations to generate translocation of the $\gamma$ peptides from the interior (under) of the capsid to the exterior (above). We performed the biasing in two separate approaches. In one approach (referred to as coupled $C V$ ) the collective COM of the five peptides was biased; in the second approach (single peptide $C V$ ), the COM of a single peptide was biased. A representative trial for each biasing method is presented in Movie S3 (coupled CV) and Movie S4 (single peptide $C V$ ) for the low $\mathrm{pH}$ system. The coupled $C V$ was partially motivated by speculation in the literature that the five peptides would move as a unit/bundle and externalize simultaneously(19, 41). Using the coupled $C V$ was an attempt to evaluate the bundle hypothesis, where if there interpeptide interactions were sufficiently strong the peptides they would move collectively (stay as a bundle) and not separate. We conducted 10 independent pulling simulations for each system (neutral and low $\mathrm{pH}$ capsid) and all simulation trajectories clearly show that $\gamma$ peptides were not liberated simultaneously. This finding contradicts the previous bundle hypothesis. We have calculated the nonequilibrium work done by the pulling force required for liberation of the first $\gamma$ peptide using 
the coupled $C V$ (Figure S2A-B). We observe that the low $\mathrm{pH}$ environment reduces the work required for externalization in a statistically significant manner (Figure S2B). We also examined the maximum force observed during the SMD trials but did not find the $\mathrm{pH}$ significantly affected this quantity (Figure S2C). For the trials at each $\mathrm{pH}$ which displayed the lowest work values, we continued the externalization until all five peptides were externalized. For this calculation, we removed the $\gamma$ peptides from the system once they were liberated from the capsid and then restarted the SMD run with the coupled $C V$ based on the remaining peptides COM. The work required to liberate all five peptides is reduced in low $\mathrm{pH}$ compared to neutral $\mathrm{pH}$ (Figure S3). Interestingly, the largest amount of work is required to release the first peptide and then the amount of work subsequently decreases as the following peptides are liberated. This results indicates the release of the first peptide is critical and possibly the rate-limiting step to achieve membrane disruption, which motivates further investigation into the mechanism and energetics of the release of a single peptide.

To further explore the $\gamma$ liberation pathway, we performed 20 independent steered MD simulations at each $\mathrm{pH}$, using the single peptide $C V$. The temporal evolution of external work done by the pulling force for the individual trajectories is shown in Figure 5A, and a clear separation
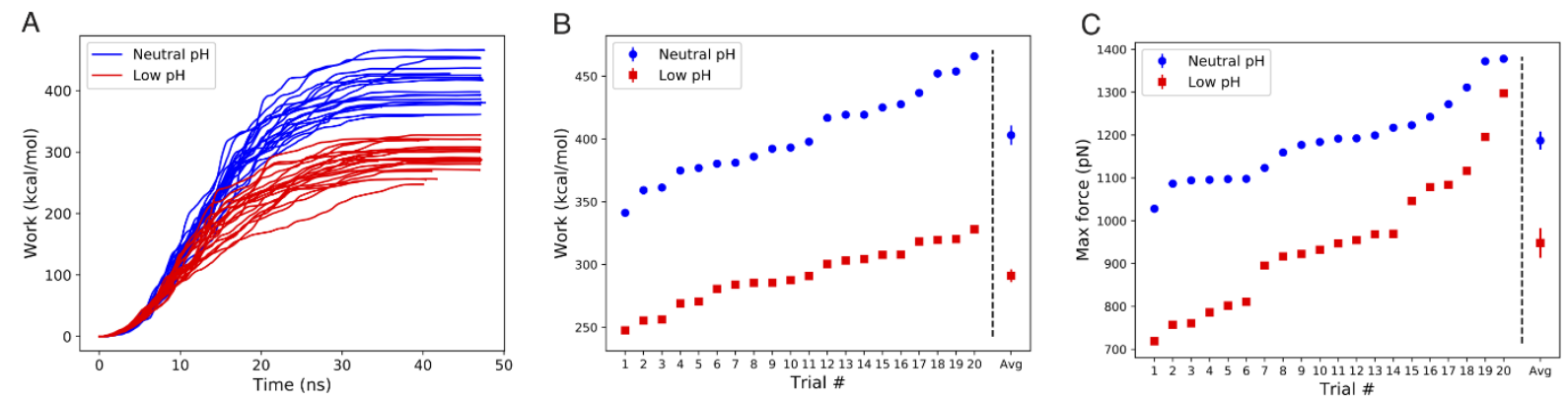

Figure 5. SMD results using single peptide $\boldsymbol{C} \boldsymbol{V}$. A) The work versus time for the 20 trials at each $\mathrm{pH}$. B) The final work values are sorted and presented from smallest to largest. The average over the trials is shown at the far right. C) The maximum force values are presented and ordered from smallest to largest. The average over the trials is shown at the far right. In (B-C) the error bars on the average values are the standard error of the means. 
(non-overlapping distribution) is observed. The final work values are compared in Figure 5B and at neutral $\mathrm{pH}$ the average work is $403( \pm 8) \mathrm{kcal} / \mathrm{mol}$ and at low $\mathrm{pH}$ the average is $291( \pm 5)$ $\mathrm{kcal} / \mathrm{mol}$, a reduction of over $100 \mathrm{kcal} / \mathrm{mol}$ when the $\mathrm{pH}$ is lowered. The SMD bias velocity was $0.1 \mathrm{~nm} / \mathrm{ns}$ in both biasing approaches (coupled and single peptide CVs). To check that the work trends are not highly sensitive to the pulling velocity a series of SMD trials were performed at different pulling rates $(0.2,0.4,0.8 \mathrm{~nm} / \mathrm{ns}$, see Figure $\mathbf{S 4})$, which demonstrate that the results are
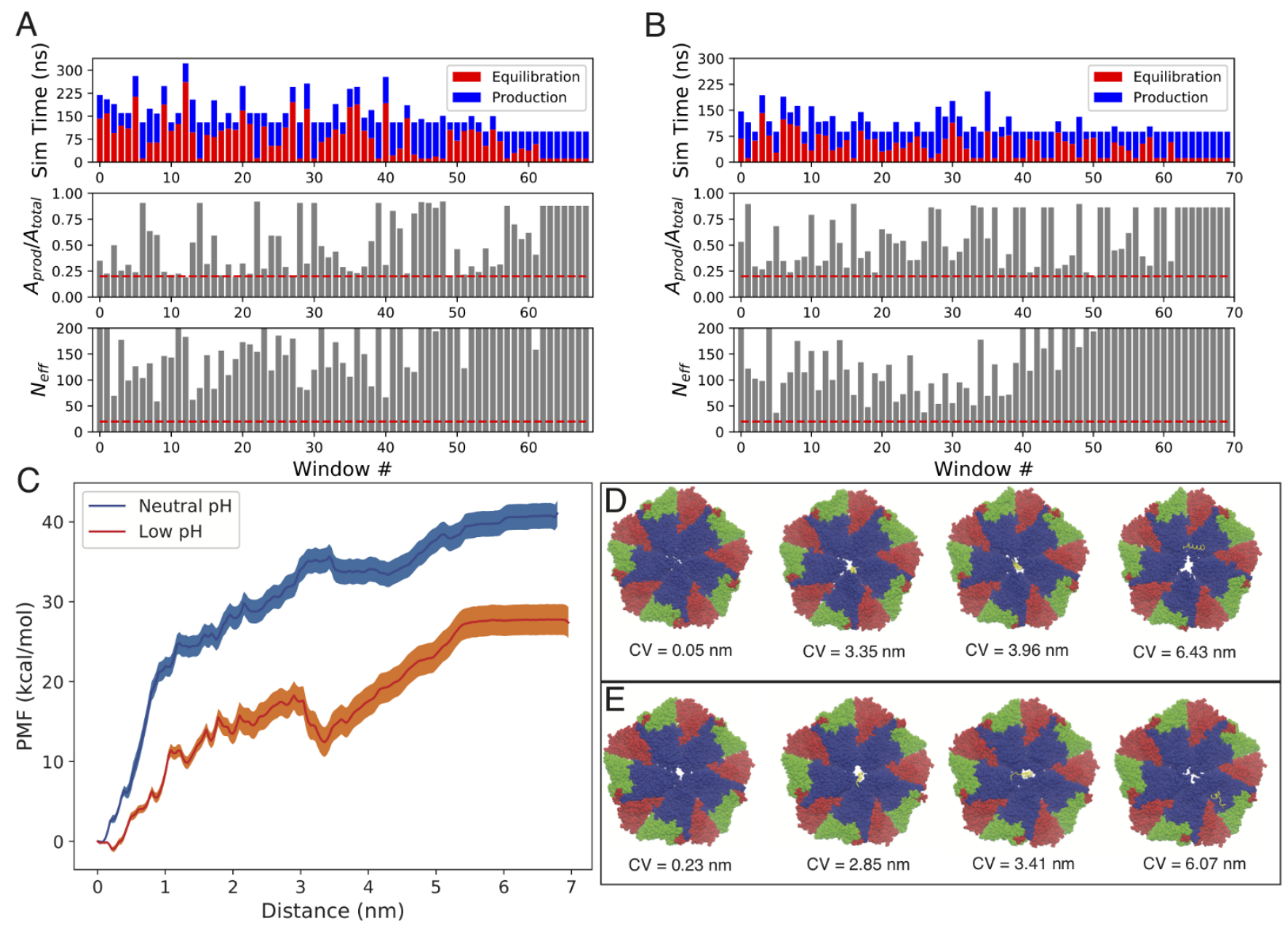

Figure 6. Energetic Analysis of $\boldsymbol{\gamma}$ liberation pathways. Time series analysis of umbrella sampling data at neutral (A) and low $\mathrm{pH}(\mathrm{B})$. The top plot shows the breakdown between equilibration and production data for each window. The middle panel shows the ratio of production to total simulation data, with dashed red line drawn at 20\%. The bottom panel shows $N_{\text {eff }}$ with dashed red line drawn at $N_{\text {eff }}=20$. The y-axis is terminated at 200, to highlight lower sampled windows, though many windows have $N_{\text {eff }}>200$. C) PMF force at neutral and low $\mathrm{pH}$. Shaded region represents the standard deviation obtain from error analysis using 1000 bootstrapped profiles. D-E) Structures along the $\gamma$ liberation pathway at neutral $\mathrm{pH}$ (D) and low $\mathrm{pH}(\mathrm{E})$ pathways. Snapshots are the final frame from a given umbrella window. 
not unique to the pulling velocity chosen. The average maximum force is significantly higher $(1187 \pm 21 \mathrm{pN})$ at neutral $\mathrm{pH}$ compared to low $\mathrm{pH}(948 \pm 35 \mathrm{pN})$, indicating that stronger (or more) interactions resist the $\gamma$ liberation process in neutral conditions(Figure 5C). The maximum forces are large, however other viruses, including $T=3$ non-enveloped capsids have been shown to be able to withstand applied forces in the range of $1000 \mathrm{pN}$ without undergoing irreversible deformation. This is based on atomic force microscopy (AFM) nanoindentation experiments and CG MD simulations.(44-47) The SMD results appear consistent with the linear mutual information analyses (Figure 4D-E), which indicated the system was more strongly correlated and rigid at neutral $\mathrm{pH}$. Hence, our multiple SMD simulations confirm that the energetic requirements to release a $\gamma$ peptide are significantly reduced under low $\mathrm{pH}$ conditions which is in qualitative agreement with the previous experimental work.(21) While the non-equilibrium work values can be related to the equilibrium free energy changes through the Jarzynski equality,(48) this method is difficult to converge and can require greater than 100 trials. $(49,50)$ Therefore we next attempted to estimate the equilibrium free energy profiles of $\gamma$ liberation by performing extensive umbrella sampling simulations along the minimum work pathways under the different $\mathrm{pH}$ conditions.

\section{pH Effects on energetic barriers for y liberation}

To calculate a free energy profile of $\gamma$ liberation under the different $\mathrm{pH}$ conditions, the lowest work trials at each pH (Figure 5A) were selected as initial pathways. These pathways were then subjected to extensive umbrella sampling simulations to allow for the systems to relax from the non-equilibrium pathways to a locally equilibrated pathway. To detect that each window had properly equilibrated and was well sampled, autocorrelation based timeseries analysis was performed.(51) Umbrella windows were run for up to $322 \mathrm{~ns}$ in order to retain approximately $20 \%$ 
(18.9\% was the minimum) of the data set and have a sufficient $(>35) N_{\text {eff }}$ in each window (Figure 6A-B). A wide range of equilibration times are observed, but we consistently observe fast equilibration times in the high numbered windows $(>60)$, where the peptide is externalized and dissociated from the capsid. Since the peptide is effectively in solution at this stage of the pathway, it is reasonable to expect these simulations would rapidly equilibrate.

The free energy profiles were computed from the equilibrated portion of data in each window, which totaled $5.0 \mu$ s at neutral $\mathrm{pH}$ (window minimum sampling time $=25 \mathrm{~ns}$ ) and $4.2 \mu \mathrm{s}$ at low $\mathrm{pH}$ (window minimum sampling time $=18 \mathrm{~ns})$. The resultant profiles (Figure $6 \mathbf{C})$ show a significant reduction in the barrier $\left(\Delta G_{b a r r}\right)$ and overall $\left(\Delta G_{e x t}\right)$ free energy change when the $\mathrm{pH}$ is reduced. At neutral $\mathrm{pH}$ a sharply increasing profile is observed in the first $1 \mathrm{~nm}$, followed by a more rugged and more gradual increase until reaching a barrier of $\sim 36 \mathrm{kcal} / \mathrm{mol}$ at $\sim 3.4 \mathrm{~nm}$. This is followed by a slight decrease in the PMF reaching a local minimum of $\sim 34 \mathrm{kcal} / \mathrm{mol}$ at $\sim 4.0 \mathrm{~nm}$. The PMF then increases reaching $\sim 40 \mathrm{kcal} / \mathrm{mol}$ when the peptide fully dissociates from the capsid around $6.0 \mathrm{~nm}$. Whereas in low $\mathrm{pH}$ a more gradual and jagged profile is observed reaching a barrier of $\sim 18 \mathrm{kcal} / \mathrm{mol}$ at $2.9 \mathrm{~nm}$. This barrier is followed by a sharp decrease in the PMF to $\sim 12$ $\mathrm{kcal} / \mathrm{mol}$, where the system reaches a metastable state at $\sim 3.4 \mathrm{~nm}$. The metastable state is shown in Figure $\mathbf{S 5}$ and is characterized by partial exposure of the $\gamma$ peptide onto the capsid exterior surface. This is followed by a gradual increase in energy to $\sim 28 \mathrm{kcal} / \mathrm{mol}$ at the fully dissociated state at $\sim 6.0 \mathrm{~nm}$. Snapshots corresponding to the initial, barrier, metastable and final states are shown in Figure 6D-E for both systems. Overall, we observe $\Delta G_{b a r r}$ is reduced by half (36 $\mathrm{kcal} / \mathrm{mol}$ at neutral, $18 \mathrm{kcal} / \mathrm{mol}$ at low $\mathrm{pH}$ ) and $\Delta G_{\text {ext }}$ is reduced from $40 \mathrm{kcal} / \mathrm{mol}$ to $28 \mathrm{kcal} / \mathrm{mol}$ when the system $\mathrm{pH}$ is reduced. 


\section{Discussion}

Several non-enveloped viruses including Rhinovirus, Hepatitis A, Reovirus, Parvovirus, Adenovirus and FHV contain membrane disrupting components of their capsids which are triggered to release/externalize under low $\mathrm{pH}$ (endosomal) conditions. $(4,5)$ However, the molecular mechanisms that underlie this process remain unclear and are challenging to address using current experimental techniques. The rapid progress in computational power and simulation algorithms now permits MD to investigate these complex biomolecular processes and can provide important insights at atomic resolution into the mechanism of action, where experimental characterization is rather difficult if not impossible.(52)

In the present study, we have investigated the mechanism of lytic peptides liberation from the FHV capsid and the role of $\mathrm{pH}$ in affecting the structural dynamics and energetics of the liberation process through a multiscale atomistic simulation approach including equilibrium simulations, steered MD and extensive umbrella sampling. Based on the FHV structure-based model, it is hypothesized that $\gamma$ peptides located under the fivefold axis are liberated from the capsid to disrupt cellular membranes and permit infection. It is known from in-vitro studies that $\gamma$ peptides disrupt membranes(13-15) and do so with maximal efficiency at $\mathrm{pH}$ 6.(21) Furthermore, when the $\gamma$ peptides are not cleaved from the capsid protein the particles are effectively noninfectious,(11) substantiating the critical role these peptides play in viral infection. Interestingly, from our $0.5 \mu$ s long equilibrium simulations we are able to observe pore opening at the fivefold axis under low $\mathrm{pH}$ conditions (Figure 3, Movie S2). Our analysis demonstrates that at low $\mathrm{pH}$, protonation of histidine residues affect (decrease) allosteric communications across the capsid, which may lead to the gate-keeper loop opening (pore formation) in the capsid (Figure 4). The role of the two histidines as "pH sensor" residues which can affect capsid dynamics is supported 
by conservation of the histidine positions in closely related nodaviruses (see multiple sequence alignment in Figure S6). Though the formation of a pore in the low $\mathrm{pH}$ capsid is an exciting observation, we do not observe spontaneously translocation of the $\gamma$ peptides, which would presumably require simulation time scales beyond our current computational capabilities.

It is therefore informative to understand how pore formation in the low $\mathrm{pH}$ capsid affect the energetics of $\gamma$ liberation process. In this study the $\gamma$ liberation process is characterized through a series of SMD runs, followed by extensive umbrella sampling along the most favorable pathways. Similar approaches combining SMD with umbrella sampling have been successfully applied to study processes of other large biological complexes such as the chromatophore(53) and membrane transporters.(54) The SMD simulation results provide a qualitative but conclusive demonstration that the work necessary for externalizing $\gamma$ peptides is distinctly lower at $\mathrm{pH} 6$ than at $\mathrm{pH}$ 7, which is well corelated with the optimal $\mathrm{pH}$ of FHV membrane lytic activity.(21) In our umbrella sampling approach we allow for long relaxation times ( $>100 \mathrm{~ns})$ and select only well equilibrated data, based upon autocorrelation analysis, to reconstruct the free energy profiles. We observe significant reductions in the barriers and overall free energy change required to externalize a peptide at low $\mathrm{pH}$.

While the overall free energy changes we observe, even at low $\mathrm{pH}$, are positive there are a few factors which could contribute to this result. One is that the initial pathways based upon SMD are far from equilibrium. Even though the umbrella sampling simulations are quite long, compared to previous all-atom studies on capsids, $(37,55)$ there could be residual strains in the system which are not fully relaxed leading to overestimation of the free energy profiles along the reaction coordinate. Another, more physical consideration, is to contemplate the closed system of the capsid within a membrane bound vesicle/endosome. The process of liberating the peptide would 
involve the free energy cost of externalizing the peptide, but also the free energy of partitioning the peptide into the membrane, which is known to be favorable. Experimental measurements indicate the $\Delta G_{\text {part }}$ for $\gamma_{1}$ partitioning into lipid bilayers to be approximately $-8 \mathrm{kcal} / \mathrm{mol}(14)$, while $\mathrm{CG}$ simulations have estimated the $\Delta \mathrm{G}$ of $\gamma_{1}$ binding to different bilayers in the range of -15 $\mathrm{kcal} / \mathrm{mol}$ to $-22.5 \mathrm{kcal} / \mathrm{mol}$, when the peptide is in a helical state.(56) This computationally computed free energy of binding is comparable to the computed free energy of externalization (28 $\mathrm{kcal} / \mathrm{mol}$ ) at low $\mathrm{pH}$. Furthermore, the peptide is partially exposed in the metastable state (Figure S5) and if the virus were to closely approach the membrane the peptide might be able to initiate contact with the bilayer before fully dissociating from the capsid. In this scenario the overall process could be favorable as the externalization free energy cost to reach the metastable state is only $12 \mathrm{kcal} / \mathrm{mol}$ at low $\mathrm{pH}$ but is far too high $(34 \mathrm{kcal} / \mathrm{mol})$ to be favorable at neutral $\mathrm{pH}$. It remains unclear if the peptides fully dissociate from the FHV capsid or if they are remaining in contact with the capsid. There would be a functional benefit for $\gamma \mathrm{s}$ to remain in contact with the capsid, as this would allow for the capsid to be proximal to the location of membrane disruption. In poliovirus there is evidence to support membrane disrupting peptides are involved in umbilical connections between the capsid and bilayer.(57)

In this work we have explicitly modeled a curvature restrained fifteen subunit subsection of the FHV capsid, which represents $1 / 12$ of the complete capsid. It is known that large biomolecular assemblies, such as virus capsid exhibit long range collective motions, which can often be low frequency (low energy) motions that can couple to functional dynamics of the systems. $(27,38,58)$ It is possible in the context of the complete capsid that collective motions would facilitate the peptide release, which are damped in our reduced system, however our LMI analysis indicates that the capsid becomes less collective at low $\mathrm{pH}$ (Figure 4). Another component 
to the complete virus, which is neglected in the current study, is the viral genome. While we cannot discount the possibility that low $\mathrm{pH}$ induces conformational changes in the genome, which then influence the pathway and energetics of $\gamma$ liberation, there is evidence that $\gamma$ release and genome release for FHV are separate events. This is based upon a recent study that used heat to trigger genome release from the FHV capsid.(59) During the heating they observe multiple transitions measured by differential scanning calorimetry (DSC) for the wild-type virus, but only a single transition for maturation defective mutants. The first transition for the wild-type particle corresponds to $\gamma$ release as detected by anti- $\gamma$ immunolabeling and is independent from the genome exposure transition.

There is increasing interest in understanding asymmetric dynamical processes in highly symmetric virus structures as these are functionally relevant processes and now both MD and cryoEM are capable of characterizing these phenomena. $(60,61)$ The present study provides the first free energy profile for membrane lytic peptide liberation from a nonenveloped virus at different $\mathrm{pH}$ values. The results provide strong evidence that lowering the $\mathrm{pH}$ induces increased fluctuations at the capsid fivefold vertex and reduces the energy requirement to externalize the lytic peptide. We identify a metastable state at low $\mathrm{pH}$ which positions the peptide in an externalized but capsid associated state. When the membrane binding step is taken into consideration the overall process of transitioning a peptide from the capsid interior to the membrane may approach a negative $\Delta \mathrm{G}$, especially if the membrane binding were to from the metastable state. Overall, our simulation study results qualitatively agrees with the previous experimental observation and elucidates several molecular-level aspects of how low $\mathrm{pH}$ can trigger capsid structural rearrangement, facilitating externalization of membrane lytic peptides that can disrupt the endosomal membrane and allow for viral infection to proliferate. 


\section{Methods System Preparation}

In this study we modeled a subsection of the complete FHV capsid, comprising five asymmetric units surrounding a fivefold symmetry axis (Figure 2A). The initial coordinates used were taken from the $2.7 \AA$ resolution X-ray crystal structure (PDB ID: 4FTB). The N-terminal tail of the A, $\mathrm{B}$ and $\mathrm{C}$ subunit, and $\mathrm{C}$-terminal regions of the $\gamma$ peptides are unstructured and not resolved in the crystal structure. The unstructured regions of the capsid, residues1-55 of chain A, residues 1-52 of chain $\mathrm{B}$, residues 1-54 of chain $\mathrm{C}$ and residues 385-407 of the $\gamma$ peptides were not modelled into this structure to avoid artifacts from generating initial conformations of large undetermined regions. Four $\mathrm{Ca}^{2+}$ ions are bound to each iASU, and these ions were included in our starting structure. To maintain the capsid curvature and mimic a full-capsid model, weak positional restraints (force constant of $500 \mathrm{~kJ} \mathrm{~mol}^{-1} \mathrm{~nm}^{-2}$ ) were applied to several $\mathrm{C}_{\alpha}$ atoms, positioned along the edge of the pentameric structure. Restrained atoms are listed and shown in Figure S7. Low $\mathrm{pH}$ conditions were modeled by protonating residues $\mathrm{D} 75, \mathrm{H} 215$ and $\mathrm{H} 334$. The low $\mathrm{pH}$ system is attempting to model $\mathrm{pH} \sim 6$ which is the environment where FHV capsids have maximal lytic activity.(21) There are only two histidine residues in each subunit and the choice to protonate the histidines is based upon assuming a $\mathrm{p} K_{\mathrm{a}} \sim 6.5$.(62) $\mathrm{D} 75$ was protonated based upon a previous $\mathrm{p} K_{\mathrm{a}}$ prediction of $\sim 6.0$ and the proposed reaction mechanism for $\gamma$ cleavage involves a protonated D75 acting as a hydrogen donor.(63) Each system was solvated with the TIP3P water model(64) and was neutralized by the addition of $\mathrm{Na}^{+}$ions and additional $\mathrm{Na}^{+}$and $\mathrm{Cl}^{-}$ions were added to generate a physiological ion concentration of $0.15 \mathrm{M} \mathrm{NaCl}$. The total system sizes were approximately 390,000 atoms. 
It is worthwhile to discuss the use of fixed protonation states in modeling different $\mathrm{pH}$ environments as we done in this work. Sophisticated methods have been developed to model constant $\mathrm{pH}$ environments in atomistic MD simulations, where residue titration states are coupled to the system dynamics. $(65,66)$ This approach has been applied to the study of virus capsid systems, $(37,67)$ however those studies were performed on a single asymmetric unit using rotational symmetry boundary conditions and in an implicit solvent environment. The constantpH MD method has been recently extended to be compatible with explicit solvent either through a hybrid implicit/explicit solvent approach(68) or through the multi-site $\lambda$ dynamics formalism.(69) While the explicit solvent constant $\mathrm{pH}$ approaches are very promising, especially for membrane-protein systems, they still come with a high computational demand. To our knowledge the application of these methods have been limited to system sizes in the range of 50,000 atoms and with individual trajectory lengths no longer than 62 ns.(70-73) In our study we are able to achieve simulations times up to $500 \mathrm{~ns}$ and total sampling for over $23 \mu$ s for an explicitly solvated system of nearly 400,000 atoms, which enables us to uncover principals that underlie acid-induced externalization of $\gamma$ peptides from the capsid and qualitatively agree with previous experimental observations.

\section{General Simulation Protocol}

All simulations in this study were performed with the GROMACS 2018 simulation package(74) using the CHARMM36 all-atom force field.(75) All simulation types, lengths and trials are listed in Table 1. The systems were first energy minimized using the steepest-descent algorithm followed by 100 ps of NVT (300 K) followed by 100 ps of NPT (300 K, 1 atm) equilibrations. In the NVT and NPT equilibrations, the nonhydrogen (heavy) atoms of the protein 
were restrained with a force constant of $1,000 \mathrm{~kJ} \mathrm{~mol}^{-1} \mathrm{~nm}^{-2}$. Production MD simulations were performed for $500 \mathrm{~ns}$ at a temperature of $300 \mathrm{~K}$ and a pressure of $1 \mathrm{~atm}$. Constant temperature was maintained using the Nose-Hoover thermostat $(76,77)$ with a coupling time constant of 1 ps and constant pressure was maintained using the Parrinello-Rahman barostat(78) with a coupling constant of 5 ps. Simulation timestep was 2 fs and waters were kept rigid using the SETTLE algorithm.(79) Non-water bonds involving hydrogen atoms were held fixed using the LINCS algorithm.(80) Electrostatic interactions were calculated by using the particle-mesh Ewald (PME) $\operatorname{method}(81)$ with a real-space cutoff of $12 \AA$. The cutoff for Van der Waal interactions was set to $12 \AA$, with smoothing starting at $10.5 \AA$.

\section{Steered Molecular Dynamics (SMD) Simulation}

Externalization of $\gamma$ peptides from the capsid were characterized through a series of steered MD (SMD) simulations, where a constant velocity pulling restraint was applied to a collective variable $(\mathrm{CV})$, which was the center of mass $(\mathrm{COM})$ of the backbone $\mathrm{C}_{\alpha}$ atoms of the $\gamma$ peptides to produce movement along the $\mathrm{z}$ axis. The biasing potential $\left(U_{S M D}\right)$ for $\mathrm{SMD}$ is:

$$
U_{S M D}(t)=\frac{1}{2} k\left[v t-\left(\mathbf{r}(t)-\mathbf{r}_{0}\right) \cdot \mathbf{n}\right]^{2}
$$

where, $k$ is the pulling force constant and $v$ is the pulling rate. $\mathbf{r}(t)$ is the position of the $\mathrm{CV}$ at time $t$ and $\mathbf{r}_{0}$ is the initial value of the $\mathrm{CV}, \mathbf{n}$ is the direction of pulling, which in this study was set as the unit vector in the Z-direction. Starting conformations for SMD simulations were randomly chosen from the last $100 \mathrm{~ns}$ of the equilibration simulations. The majority of simulations used a pulling velocity of $0.1 \mathrm{~nm} / \mathrm{ns}$ and the pulling force constant was $1,000 \mathrm{~kJ} \mathrm{~mol}^{-1} \mathrm{~nm}^{-2}$. Two different CVs were examined, $\mathrm{CV}_{1}$ (coupled), in which the $\mathrm{COM}$ position of all five peptides was collectively biased and $\mathrm{CV}_{2}$ (single peptide) where a single peptide was chosen and it's COM 
position was biased. The non-equilibrium work $(W(t))$ is calculated by integrating the timedependent force $(F(t))$ acting on the biased $\mathrm{CV}$,

$$
W(t)=\int F(t) d t
$$

using the cumtrapz function in MATLAB. It is worthwhile to mention that the non-equilibrium work profiles derived from SMD simulations are dominated by the dissipative energy component which is stochastic in nature. Therefore, we performed 40 SMD simulations for each system using different $\mathrm{CVs}$, different starting conformations and different pulling velocities (0.1-0.8 $\mathrm{nm} / \mathrm{ns}$, Figure S4) to enable reliable observations of these systems.

\section{Umbrella Sampling Simulation}

We have performed umbrella sampling simulations(82-84) using GROMACS 2018, to estimate the free energy change associated with $\gamma$ liberation from the capsid at neutral and low $\mathrm{pH}$ . A harmonic umbrella potential (force constant of $2092 \mathrm{~kJ} \mathrm{~mol}^{-1} \mathrm{~nm}^{-2}$ ) was used to restrain the COM of a single liberating $\gamma$ peptide along $\mathrm{z}$ axis. Umbrella windows were spaced $1 \AA$ apart and spanned the entire $\gamma$ liberation pathway $(\sim 7 \mathrm{~nm})$, resulting in 69 windows for the neutral system and 70 windows for the low $\mathrm{pH}$ system. Each umbrella window was sampled for at least $88 \mathrm{~ns}$, amounting to a total of $18 \mu$ s simulation time. To improve the estimate of the potential of mean force, $(\mathrm{PMF})$ we have extracted only equilibrated samples from each window using the timeseries module of the pymbar package.(51) We have run each umbrella window until approximately $20 \%$ of the data is selected as equilibrium samples and there are at least 35 effectively uncorrelated samples $\left(N_{\text {eff }}\right)$ for each window. $N_{\text {eff }}$ is defined as

$$
N_{e f f}=\frac{T-t_{0}+1}{1+2 \tau_{a c}}
$$


where $T$ is the total simulation length, $t_{0}$ is the equilibration time and $\tau_{\mathrm{ac}}$ the decorrelation time of the biased CV.(51) A minimum equilibration time of $12 \mathrm{~ns}$ was applied to windows with $t_{0}$ less than $12 \mathrm{~ns}$. The unbiased free energy surface was constructed from the umbrella histograms, using the weighted histogram analysis method (WHAM)(85) implemented in GROMACS gmx wham tool(86). All umbrella histograms were visually inspected to ensure significant overlap between the histograms from adjacent simulation windows. The PMFs were converged at $10^{-6} \mathrm{kcal} \mathrm{mol}^{-1}$ tolerance. We have estimated the statistical uncertainty of our free energy profile using Bayesian bootstrap of complete histograms method, freely distributed with the GROMACS gmx wham tool(86) and 1000 bootstraps were generated to calculate the statistical error.

\section{Trajectory Analysis}

Root-mean-square-deviation (RMSD) of the capsid $\mathrm{C}_{\alpha}$ atoms was calculated with respect to its initial state using the GROMACS tool gmx rms. Translational and rotational superposition of the capsid with respect to its initial state is performed, prior to RMSD calculation. Hydrophobic solvent accessible surface area (SASA) was calculated using the VMD package(87) by using a spherical probe of $1.8 \AA$. The definition of hydrophobic residues for this calculation is Ala, Leu, Val, Ile, Pro, Phe, Met and Trp. The calculation was performed over the A, B and C subunits but did not include the $\gamma$ peptides. The final $300 \mathrm{~ns}$ of each of the equilibrium simulations at each $\mathrm{pH}$ (900 ns total at each $\mathrm{pH}$ ) was analyzed. Analysis of the pore radius at the fivefold vertex was performed on the capsid over the multiple equilibrium simulation trajectories using the program HOLE(88), implemented in MDAnalysis(89). This pore calculation was performed every $1 \mathrm{~ns}$ over the course of the multiple 500 ns equilibrium trajectories at each $\mathrm{pH}$. Residue-wise secondary 
structural propensity of the capsid subunit A protein was computed using the Dictionary of Secondary Structure in Proteins (DSSP) implemented in GROMACS do_dssp tool.

Mutual Information quantifies the amount of information in one unknown variable $\left(X_{j}\right)$ obtained from the state of another known variable $\left(X_{i}\right)$. We have calculated the linear contribution (LMI) $\left(I_{\text {lin }}\left(X_{i}, X_{j}\right)\right)$ using bio3d $(42,43)$ and based on the following formula:

$$
I_{l i n}\left(\mathbf{x}_{i}, \mathbf{x}_{j}\right)=\frac{1}{2}\left[\ln \operatorname{det} \mathbf{C}_{(i)}+\ln \operatorname{det} \mathbf{C}_{(j)}+\ln \operatorname{det} \mathbf{C}_{(i j)}\right]
$$

where $\mathbf{C}_{i}=\left\langle\mathbf{x}_{i}^{T} \mathbf{x}_{i}\right\rangle$ is the marginal covariance matrix, $\mathbf{C}_{i j}=\left(\left\langle\mathbf{x}_{i} \mathbf{x}_{j}\right\rangle^{T}\left\langle\mathbf{x}_{i} \mathbf{x}_{j}\right\rangle\right)$ is the pair covariance matrix and $\mathbf{x}_{i}$ is the positional fluctuation of the C $\alpha$ atom of residue $i$. Unlike, general mutual information analysis, this method does not require a computationally expensive entropy calculation and provides a lower bound on the mutual information estimate. For both the secondary structure and the LMI analyses the data was averaged over $4.5 \mu$ s of data at each $\mathrm{pH}$. This amount of data arises from using the last $300 \mathrm{~ns}$ of each of the three trials and averaging over the five A subunits in our simulation system.

Table 1. List of simulation types and lengths.

\begin{tabular}{|l|c|c|c|c|}
\hline \multicolumn{1}{|c|}{ Type } & pH & Trials/Windows & $\begin{array}{c}\text { Simulation } \\
\text { Length }\end{array}$ & $\begin{array}{c}\text { Total Simulation } \\
\text { Time }\end{array}$ \\
\hline Equilibrium & Neutral & 3 & $500 \mathrm{~ns}$ & $1.50 \mu \mathrm{s}$ \\
\hline Equilibrium & Low & 3 & $500 \mathrm{~ns}$ & $1.50 \mu \mathrm{s}$ \\
\hline SMD - Single Peptide CV & Neutral & 20 & $25-48 \mathrm{~ns}$ & $945 \mathrm{~ns}$ \\
\hline SMD - Single Peptide CV & Low & 20 & $34-47 \mathrm{~ns}$ & $889 \mathrm{~ns}$ \\
\hline SMD - Coupled CV & Neutral & 10 & $27-41 \mathrm{~ns}$ & $334 \mathrm{~ns}$ \\
\hline SMD - Coupled CV & Low & 10 & $22-34 \mathrm{~ns}$ & $284 \mathrm{~ns}$ \\
\hline Umbrella Sampling & Neutral & 69 & $100-322 \mathrm{~ns}$ & $10.67 \mu \mathrm{s}$ \\
\hline Umbrella Sampling & Low & 70 & $88-206 \mathrm{~ns}$ & $7.51 \mu \mathrm{s}$ \\
\hline All Trajectories & & & & $23.63 \mu \mathrm{s}$ \\
\hline
\end{tabular}

\section{ACKNOWLEDGMENTS}


We would like to thank Kevin Boyd for providing guidance and scripts for the timeseries analysis. We also would like to acknowledge Allyn Brice, Michael Ward and Ehsan Moharreri for their efforts working on this project in earlier stages. This research has been supported by the National Institutes of Health through grant number R35-GM119762 (to E.R.M.). This work used the Extreme Science and Engineering Discovery Environment (XSEDE) for HPC resources at the Texas Advanced Computing Center (TACC) through allocation TG-MCB140016. Additional computational resources for this work have been provided through the University of Connecticut Storrs HPC center.

\section{References}

1. W. Weissenhorn, A. Hinz, Y. Gaudin, Virus membrane fusion. FEBS Letters 581, 2150-2155 (2007).

2. S. C. Harrison, Viral membrane fusion. Nature structural \& molecular biology 15, 690-698 (2008).

3. M. Banerjee, J. E. Johnson, Activation, exposure and penetration of virally encoded, membrane-active polypeptides during non-enveloped virus entry. Current Protein and Peptide Science 9, 16-27 (2008).

4. C. S. Kumar, D. Dey, S. Ghosh, M. Banerjee, Breach : Host Membrane Penetration and Entry by Nonenveloped Viruses. Trends in Microbiology xx, 1-13 (2017).

5. C. L. Moyer, G. R. Nemerow, Viral weapons of membrane destruction: Variable modes of membrane penetration by non-enveloped viruses. Current Opinion in Virology (2011) https:/doi.org/10.1016/j.coviro.2011.05.002.

6. B. Tsai, Penetration of Nonenveloped Viruses into the Cytoplasm. 23-43 (2007).

7. A. Odegard, M. Banerjee, J. E. Johnson, Flock house virus: A model system for understanding non-enveloped virus entry and membrane penetration. Current Topics in Microbiology and Immunology 343, 1-22 (2010).

8. T. M. Gallagher, R. R. Rueckert, Assembly-dependent maturation cleavage in provirions of a small icosahedral insect ribovirus. Journal of virology 62, 3399-3406 (1988). 
9. B. H. Selling, R. F. Allison, P. Kaesberg, Genomic RNA of an insect virus directs synthesis of infectious virions in plants. Proceedings of the National Academy of Sciences of the United States of America 87, 434-438 (1990).

10. B. D. Price, M. Roeder, P. Ahlquist, DNA-Directed expression of functional flock house virus RNA1 derivatives in Saccharomyces cerevisiae, heterologous gene expression, and selective effects on subgenomic mRNA synthesis. Journal of virology 74, 11724-11733 (2000).

11. A. Schneemann, W. Zhong, T. M. Gallagher, R. R. Rueckert, Maturation Cleavage Required for Infectivity of a Nodavirus. 66, 6728-6734 (1992).

12. A. J. Fisher, J. E. Johnson, Ordered duplex RNA controls capsid architecture in an icosahedral animal virus. Nature 361, 176-179 (1993).

13. D. T. Bong, C. Steinem, A. Janshoff, J. E. Johnson, M. Reza Ghadiri, A highly membraneactive peptide in Flock House virus: implications for the mechanism of nodavirus infection. Chemistry \& biology 6, 473-481 (1999).

14. D. T. Bong, A. Janshoff, C. Steinem, M. R. Ghadiri, Membrane partitioning of the cleavage peptide in flock house virus. Biophysical journal 78, 839-845 (2000).

15. L. F. Maia, et al., Structure of a membrane-binding domain from a non-enveloped animal virus: Insights into the mechanism of membrane permeability and cellular entry. Journal of Biological Chemistry 281, 29278-29286 (2006).

16. A. Schneemann, D. Marshall, Specific encapsidation of nodavirus RNAs is mediated through the $C$ terminus of capsid precursor protein alpha. Journal of virology $72,8738-$ 8746 (1998).

17. M. Banerjee, et al., Dissecting the Functional Domains of a Nonenveloped Virus Membrane Penetration Peptide. Journal of Virology 83, 6929-6933 (2009).

18. S. Nangia, K. J. Boyd, E. R. May, Molecular dynamics study of membrane permeabilization by wild-type and mutant lytic peptides from the non-enveloped Flock House virus. Biochimica et Biophysica Acta (BBA) - Biomembranes 1862, 183102 (2020).

19. R. Holland Cheng, et al., Functional implications of quasi-equivalence in a $T=3$ icosahedral animal virus established by cryo-electron microscopy and X-ray crystallography. Structure (1994) https:/doi.org/10.1016/S0969-2126(00)00029-0.

20. A. J. Fisher, J. E. Johnson, Ordered duplex RNA controls capsid architecture in an icosahedral animal virus. Nature 361, 176-179 (1993).

21. A. L. Odegard, et al., Low endocytic $\mathrm{pH}$ and capsid protein autocleavage are critical components of flock house virus cell entry. Journal of Virology 83, 8628-8637 (2009). 
22. T. Domitrovic, T. Matsui, J. E. Johnson, Dissecting Quasi-Equivalence in Nonenveloped Viruses: Membrane Disruption Is Promoted by Lytic Peptides Released from Subunit Pentamers, Not Hexamers. Journal of Virology 86, 9976 LP - 9982 (2012).

23. T. Matsui, G. C. Lander, R. Khayat, J. E. Johnson, Subunits fold at position-dependent rates during maturation of a eukaryotic RNA virus. Proceedings of the National Academy of Sciences of the United States of America 107, 14111-14115 (2010).

24. B. Bothner, X. F. Dong, L. Bibbs, J. E. Johnson, G. Siuzdak, Evidence of viral capsid dynamics using limited proteolysis mass spectrometry. Journal of Biological Chemistry 273, 673-676 (1998).

25. R. O. Dror, R. M. Dirks, J. P. Grossman, H. Xu, D. E. Shaw, Biomolecular Simulation: A Computational Microscope for Molecular Biology. Annual Review of Biophysics (2012) https:/doi.org/10.1146/annurev-biophys-042910-155245.

26. E. H. Lee, J. Hsin, M. Sotomayor, G. Comellas, K. Schulten, Discovery Through the Computational Microscope. Structure 17, 1295-1306 (2009).

27. J. A. Hadden, et al., All-atom molecular dynamics of the HBV capsid reveals insights into biological function and cryo-EM resolution limits. Elife 7, e32478 (2018).

28. D. S. D. Larsson, L. Liljas, D. van der Spoel, Virus capsid dissolution studied by microsecond molecular dynamics simulations. PLoS computational biology 8, e1002502-e1002502 (2012).

29. Y. Andoh, et al., All-atom molecular dynamics calculation study of entire poliovirus empty capsids in solution. The Journal of Chemical Physics 141, 165101 (2014).

30. E. Tarasova, et al., All-Atom Molecular Dynamics Simulations of Entire Virus Capsid Reveal the Role of Ion Distribution in Capsid's Stability. The Journal of Physical Chemistry Letters 8, 779-784 (2017).

31. O. M. Elrad, M. F. Hagan, Encapsulation of a polymer by an icosahedral virus. Phys. Biol. 7, 045003 (2010).

32. J. D. Perlmutter, C. Qiao, M. F. Hagan, Viral genome structures are optimal for capsid assembly. elife 2, e00632-e00632 (2013).

33. H. D. Nguyen, C. L. Brooks III, Generalized Structural Polymorphism in Self-Assembled Viral Particles. Nano Lett. 8, 4574-4581 (2008).

34. H. D. Nguyen, V. S. Reddy, C. L. Brooks III, Invariant Polymorphism in Virus Capsid Assembly. J. Am. Chem. Soc. 131, 2606-2614 (2009). 
35. D. C. Rapaport, Molecular dynamics simulation of reversibly self-assembling shells in solution using trapezoidal particles. 86, 051917 (2012).

36. J. M. A. Grime, G. A. Voth, Early Stages of the HIV-1 Capsid Protein Lattice Formation. Biophysical Journal 103, 1774-1783 (2012).

37. E. R. May, K. Arora, C. L. Brooks III, pH-Induced Stability Switching of the Bacteriophage HK97 Maturation Pathway. J. Am. Chem. Soc. 136, 3097-3107 (2014).

38. E. R. May, J. Feng, C. L. Brooks III, Exploring the symmetry and mechanism of virus capsid maturation via an ensemble of pathways. Biophys J 102, 606-612 (2011).

39. F. Tama, C. L. Brooks III, Diversity and Identity of Mechanical Properties of Icosahedral Viral Capsids Studied with Elastic Network Normal Mode Analysis. Journal of Molecular Biology 345, 299-314 (2005).

40. M. K. Kim, R. L. Jernigan, G. S. Chirikjian, An elastic network model of HK97 capsid maturation. Journal of Structural Biology 143, 107-117 (2003).

41. S. Bajaj, D. Dey, R. Bhukar, M. Kumar, M. Banerjee, Non-Enveloped Virus Entry: Structural Determinants and Mechanism of Functioning of a Viral Lytic Peptide. Journal of Molecular Biology 428, 3540-3556 (2016).

42. B. J. Grant, A. P. C. Rodrigues, K. M. ElSawy, J. A. McCammon, L. S. D. Caves, Bio3d: an R package for the comparative analysis of protein structures. Bioinformatics 22, 2695-2696 (2006).

43. O. F. Lange, H. Grubmüller, Generalized correlation for biomolecular dynamics. Proteins: Structure, Function, and Bioinformatics 62, 1053-1061 (2006).

44. M. Castellanos, R. Pérez, P. J. P. Carrillo, P. J. de Pablo, M. G. Mateu, Mechanical Disassembly of Single Virus Particles Reveals KineticIntermediates Predicted by Theory. Biophysj 102, 2615-2624 (2012).

45. J. P. Michel, et al., Nanoindentation studies of full and empty viral capsids and the effects of capsid protein mutations on elasticity and strength. Proceedings of the National Academy of Sciences 103, 6184-6189 (2006).

46. M. Baclayon, et al., Prestress Strengthens the Shell of Norwalk Virus Nanoparticles. Nano Lett. 11, 4865-4869 (2011).

47. K. J. Boyd, P. Bansal, J. Feng, E. R. May, Stability of Norwalk Virus Capsid Protein Interfaces Evaluated by in Silico Nanoindentation. Front Bioeng Biotechnol 3, 103 (2015).

48. C. Jarzynski, Nonequilibrium Equality for Free Energy Differences. Physical Review Letters 78, 2690-2693 (1997). 
49. S. Park, F. Khalili-Araghi, E. Tajkhorshid, K. Schulten, Free energy calculation from steered molecular dynamics simulations using Jarzynski's equality. J. Chem. Phys. 119, 3559 (2003).

50. M. A. Cuendet, O. Michielin, Protein-Protein Interaction Investigated by Steered Molecular Dynamics: The TCR-pMHC Complex. Biophysical Journal 95, 3575-3590 (2008).

51. J. D. Chodera, A Simple Method for Automated Equilibration Detection in Molecular Simulations (2016) https:/doi.org/10.1021/acs.jctc.5b00784.

52. S. A. Hollingsworth, R. O. Dror, Molecular Dynamics Simulation for All. Neuron 99, 11291143 (2018).

53. A. Singharoy, et al., Atoms to Phenotypes: Molecular Design Principles of Cellular Energy Metabolism. Cell 179, 1098-1111.e23 (2019).

54. M. Moradi, E. Tajkhorshid, Mechanistic picture for conformational transition of a membrane transporter at atomic resolution. Proceedings of the National Academy of Sciences 110, 18916-18921 (2013).

55. A. Díaz-Valle, J. M. Falcón-González, M. Carrillo-Tripp, Hot Spots and Their Contribution to the Self-Assembly of the Viral Capsid: In Silico Prediction and Analysis. International Journal of Molecular Sciences 20, 5966 (2019).

56. S. Nangia, E. R. May, Influence of membrane composition on the binding and folding of a membrane lytic peptide from the non-enveloped flock house virus. BBA - Biomembranes 1859, 1190-1199 (2017).

57. M. Strauss, H. C. Levy, M. Bostina, D. J. Filman, J. M. Hogle, RNA Transfer from Poliovirus 135S Particles across Membranes Is Mediated by Long Umbilical Connectors. J Virol 87, 3903-3914 (2013).

58. J. R. Perilla, K. Schulten, Physical properties of the HIV-1 capsid from all-atom molecular dynamics simulations. Nature Communications 8 (2017).

59. K. Azad, M. Banerjee, Structural Dynamics of Nonenveloped Virus Disassembly Intermediates. Journal of Virology 93, e01115-19 (2019).

60. M. J. Conley, D. Bhella, Asymmetric analysis reveals novel virus capsid features. Biophysical Reviews 11, 603-609 (2019).

61. A. K. Jana, E. R. May, Structural and dynamic asymmetry in icosahedrally symmetric virus capsids. Current Opinion in Virology 45, 8-16 (2020).

62. C. N. Pace, G. R. Grimsley, J. M. Scholtz, Protein ionizable groups: pK values and their contribution to protein stability and solubility. Journal of Biological Chemistry 284, 1328513289 (2009). 
63. A. Zlotnick, et al., Capsid assembly in a family of animal viruses primes an autoproteolytic maturation that depends on a single aspartic acid residue. J. Biol. Chem. 269, 1368013684 (1994).

64. W. L. Jorgensen, J. Chandrasekhar, J. D. Madura, R. W. Impey, M. L. Klein, Comparison of simple potential functions for simulating liquid water. The Journal of Chemical Physics 79, 926-935 (1983).

65. J. Mongan, D. A. Case, J. A. McCammon, Constant pH molecular dynamics in generalized Born implicit solvent. Journal of Computational Chemistry (2004) https:/doi.org/10.1002/jcc.20139.

66. J. Khandogin, C. L. Brooks III, Constant pH molecular dynamics with proton tautomerism. Biophys J 89, 141-157 (2005).

67. X. Zeng, S. Mukhopadhyay, C. L. Brooks III, Residue-level resolution of alphavirus envelope protein interactions in pH-dependent fusion. P Natl Acad Sci Usa, 201414190 (2015).

68. J. A. Wallace, J. K. Shen, Continuous Constant pH Molecular Dynamics in Explicit Solvent with pH-Based Replica Exchange. J. Chem. Theory Comput. 7, 2617-2629 (2011).

69. J. L. Knight, C. L. Brooks, Multisite $\lambda$ Dynamics for Simulated Structure-Activity Relationship Studies. Journal of Chemical Theory and Computation 7, 2728-2739 (2011).

70. A. Panahi, C. L. Brooks III, Membrane Environment Modulates the $p$ KaValues of Transmembrane Helices. J Phys Chem B 119, 4601-4607 (2015).

71. J. A. Henderson, R. C. Harris, C.-C. Tsai, J. Shen, How Ligand Protonation State Controls Water in Protein-Ligand Binding. The Journal of Physical Chemistry Letters 9, 5440-5444 (2018).

72. C.-C. Tsai, Z. Yue, J. Shen, How Electrostatic Coupling Enables Conformational Plasticity in a Tyrosine Kinase. Journal of the American Chemical Society 141, 15092-15101 (2019).

73. H. Torabifard, A. Panahi, C. L. Brooks, M2 amphipathic helices facilitate $\mathrm{pH}$-dependent conformational transition in influenza A virus. Proceedings of the National Academy of Sciences 117, 3583-3591 (2020).

74. M. J. Abraham, et al., GROMACS: High performance molecular simulations through multilevel parallelism from laptops to supercomputers. SoftwareX 1-2, 19-25 (2015).

75. R. B. Best, et al., Optimization of the Additive CHARMM All-Atom Protein Force Field Targeting Improved Sampling of the Backbone $\varphi, \psi$ and Side-Chain $\chi 1$ and $\chi 2$ Dihedral Angles. Journal of Chemical Theory and Computation 8, 3257-3273 (2012). 
76. W. G. Hoover, Canonical dynamics: Equilibrium phase-space distributions. Physical Review A 31, 1695-1697 (1985).

77. S. Nosé, A unified formulation of the constant temperature molecular dynamics methods. The Journal of Chemical Physics 81, 511-519 (1984).

78. M. Parrinello, A. Rahman, Polymorphic transitions in single crystals: A new molecular dynamics method. Journal of Applied Physics 52, 7182-7190 (1981).

79. S. Miyamoto, P. A. Kollman, Settle: An analytical version of the SHAKE and RATTLE algorithm for rigid water models. Journal of Computational Chemistry 13, 952-962 (1992).

80. B. Hess, H. Bekker, H. J. C. Berendsen, J. G. E. M. Fraaije, LINCS: A linear constraint solver for molecular simulations. Journal of Computational Chemistry 18, 1463-1472 (1997).

81. U. Essmann, et al., A smooth particle mesh Ewald method. The Journal of Chemical Physics 103, 8577-8593 (1995).

82. G. N. Patey, J. P. Valleau, The free energy of spheres with dipoles: Monte Carlo with multistage sampling. Chemical Physics Letters 21, 297-300 (1973).

83. G. M. Torrie, J. P. Valleau, Monte Carlo free energy estimates using non-Boltzmann sampling: Application to the sub-critical Lennard-Jones fluid. Chemical Physics Letters 28, 578-581 (1974).

84. G. M. Torrie, J. P. Valleau, Nonphysical sampling distributions in Monte Carlo free-energy estimation: Umbrella sampling. Journal of Computational Physics 23, 187-199 (1977).

85. S. Kumar, J. M. Rosenberg, D. Bouzida, R. H. Swendsen, P. A. Kollman, THE weighted histogram analysis method for free-energy calculations on biomolecules. I. The method. Journal of Computational Chemistry 13, 1011-1021 (1992).

86. J. S. Hub, B. L. de Groot, D. van der Spoel, g_wham-A Free Weighted Histogram Analysis Implementation Including Robust Error and Autocorrelation Estimates. Journal of Chemical Theory and Computation 6, 3713-3720 (2010).

87. W. Humphrey, A. Dalke, K. Schulten, VMD: Visual molecular dynamics. Journal of Molecular Graphics 14, 33-38 (1996).

88. O. S. Smart, J. G. Neduvelil, X. Wang, B. A. Wallace, M. S. P. Sansom, HOLE: A program for the analysis of the pore dimensions of ion channel structural models. Journal of Molecular Graphics 14, 354-360 (1996).

89. N. Michaud-Agrawal, E. J. Denning, T. B. Woolf, O. Beckstein, MDAnalysis: A toolkit for the analysis of molecular dynamics simulations. Journal of Computational Chemistry 32, 23192327 (2011). 
bioRxiv preprint doi: https://doi.org/10.1101/2021.01.07.425769; this version posted January 8, 2021. The copyright holder for this preprint (which was not certified by peer review) is the author/funder, who has granted bioRxiv a license to display the preprint in perpetuity. It is made available under aCC-BY-NC-ND 4.0 International license. 